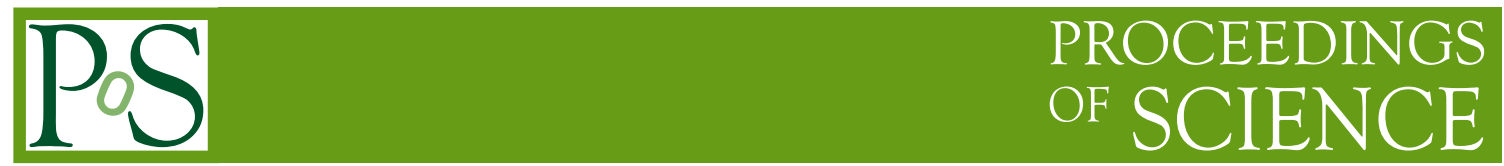

\title{
BlackHawk: A tool for computing Black Hole evaporation
}

\author{
Jérémy Auffinger ${ }^{a, *}$ and Alexandre Arbey ${ }^{a, b, c}$ \\ ${ }^{a}$ Univ Lyon, Univ Claude Bernard Lyon 1, CNRS/IN2P3, IP2I Lyon, UMR 5822, F-69622, Villeurbanne, \\ France \\ ${ }^{b}$ Institut Universitaire de France (IUF), 103 boulevard Saint-Michel, 75005 Paris, France \\ ${ }^{c}$ Theoretical Physics Department, CERN, CH-1211 Geneva 23, Switzerland
}

E-mail: j.auffinger@ipnl.in2p3.fr, alexandre.arbey@ens-lyon.fr

We present the public code BlackHawk, a powerful tool to compute the evaporation of any distribution of Schwarzschild and Kerr Black Holes and the emission spectra of Hawking radiation.

Tools for High Energy Physics and Cosmology - TOOLS2020

2-6 November, 2020

Institut de Physique des 2 Infinis (IP2I), Lyon, France

${ }^{*}$ Speaker 


\section{Introduction}

The nature of dark matter (DM) is a long standing problem in modern cosmology. For now, particle physics candidates explaining the missing mass have not been observed, neither in direct (see e.g. [1] for an overview) nor indirect searches (see e.g. [2] for a recent review). On the other hand, the concept of primordial Black Holes (PBHs) which originates from the 60s [3] has raised new interest in the last decade. The recent detection of gravitational waves from the mergers of binary BHs by the LIGO/Virgo Collaborations [4] and the observation of the shadow of the supermassive $\mathrm{BH}$ at the center of M87 [5] have shown that BHs are ubiquitous in our universe. The observed BHs are either of stellar mass or supermassive. There is a never-ending discussion about their origin: most, or at least some of them may be primordial. Many astrophysical constraints limit the fraction of DM that PBHs could represent, see for example [6] and references therein for a long review, and [7] for a more concise one. Recent work focuses on the nature of LIGO/Virgo BHs and the discrimination between the primordial and stellar origin using mass distribution or spin measurements [8-14]. Most of those studies consider PBHs with very low spin formed during the radiation domination era, but it has been shown that PBHs could have a very high spin if formed during a transient matter dominated era, evading Thorne's limit [15].

The most striking characteristics of BHs is that they should emit radiation close to the blackbody one and thus slowly evaporate away by losing mass, angular momentum and - putative - electric charge. This theoretical phenomenon first described by Hawking [16] led to the idea that BHs could be detected via the radiation they emit when evaporating [17]. For now, no experimental evidence has confirmed its existence. Hawking radiation is sizeable only for small BHs of less than $\sim 10^{16}-10^{19} \mathrm{~g}$, leading to radiation in the $\mathrm{MeV}-\mathrm{keV}$ band. This points directly towards PBHs since stellar mass BHs are heavier than $\sim 2 M_{\odot}$ due to the Tolman-Oppenheimer-Volkoff limit.

PBHs may have formed in the early universe through the collapse of strong density inhomogeneities $\Delta \rho / \rho \gtrsim 1$ resulting from quantum fluctuations during inflation, from the collapses of topological defects (cosmic strings, domain walls), or from bubble collisions during a first-order phase transition (see [6] for an extended discussion). The mass of PBHs formed in the early universe is linked to the content of the Hubble radius at the time of formation

$$
M_{\mathrm{PBH}}^{\mathrm{init}}(t) \sim 10^{15}\left(\frac{t}{10^{-23} \mathrm{~s}}\right) \mathrm{g},
$$

which corresponds approximately to the Hubble mass at time $t$. Evaporation equations show that (non-rotating) BHs have a lifetime linked to their mass by

$$
\tau\left(M_{\mathrm{PBH}}^{\mathrm{init}}\right) \sim 10^{64}\left(\frac{M_{\mathrm{PBH}}^{\mathrm{init}}}{M_{\odot}}\right)^{3} \mathrm{yr} .
$$

Thus PBHs with a mass $M_{\mathrm{PBH}}^{\mathrm{init}} \lesssim 10^{15} \mathrm{~g}$ have completely evaporated by now, thus they cannot represent a sizeable fraction of DM (existing today). These PBHs may have however left imprints of their evaporation in cosmological observations. Heavier PBHs, on the other hand, are almost eternal compared to the age of the universe due to the cubic dependence in Eq. (2). If they are of asteroid mass $M_{\mathrm{PBH}}^{\mathrm{init}} \lesssim 10^{19} \mathrm{~g}$, then their Hawking radiation may be detected by astrophysical observation and they could represent a fraction, if not all, of DM. 
The above discussion shows that there is a need for constraints on PBH DM linked to observations in order to infirm or confirm this scenario. Alternatively, mixed models assuming the existence of more than one DM component (e.g. WIMPs and PBHs [18]) can also be constrained using Hawking radiation. For now, there still exists an open window $10^{17} \mathrm{~g} \lesssim M_{\mathrm{PBH}} \lesssim 10^{21} \mathrm{~g}$ in the asteroid and sublunar mass range for PBHs to represent all of DM [6]. PBHs are also quite unconstrained in the lowest possible mass range $10^{-1} \mathrm{~g} \lesssim M_{\mathrm{PBH}} \lesssim 10^{9} \mathrm{~g}$ as there is no known cosmological observable from those times, although gravitational astronomy may soon provide new possibilities ${ }^{1}$ [20-22] (ref. [20] already studied the effect on spin on the constraints). This is the general context in which the public tool BlackHawk [23] has been created.

In section 2, we rapidly present the fundamental equations of Hawking radiation, in section 3 we discuss in some detail the capabilities of BlackHawk, in section 4 we present some examples of the work that can be done with BlackHawk and we conclude in section 5 .

\section{Hawking radiation}

Hawking radiation [16] is a semi-classical phenomenon by which pairs of particles and antiparticles are created in the vicinity of the horizon of a $\mathrm{BH}$ and can get causally separated by this horizon, resulting in a net flux of energy at spatial infinity. The creation of pairs at the horizon is a purely gravitational phenomenon whose thermodynamics is directed by the effective temperature of the $\mathrm{BH}^{2}$

$$
T \equiv \frac{\kappa}{2 \pi},
$$

where $\kappa$ is the surface gravity. Thus, the rate of Hawking radiation is simply given by the blackbody law

$$
\frac{\mathrm{d}^{2} N_{i}}{\mathrm{~d} t \mathrm{~d} E}=\sum_{\text {dof }} \frac{\Gamma_{i}\left(E, M, x_{j}\right) / 2 \pi}{e^{E^{\prime}\left(E, x_{j}\right) / T}-(-1)^{2 s_{i}}},
$$

where

- $\mathrm{d}^{2} N_{i} / \mathrm{d} t \mathrm{~d} E$ is the number of particles of type $i$ emitted per unit time $t$ and energy $E$;

- $\Gamma_{i}$ is the greybody factor describing the probability that a particle generated at the horizon escapes at infinity;

- $E^{\prime}$ is the total energy of the particles corrected for e.g. horizon rotation or electric charge;

- $s_{i}$ is the particle spin;

- $x_{j}$ are all the internal degrees of freedom of the $\mathrm{BH}$, namely angular momentum $a^{*}$ and charge $Q$ following the no-hair theorem;

- the sum runs over the internal degrees of freedom of the particles (helicity, color, etc).

${ }^{1}$ The Higgs vacuum decay triggered by PBHs brings another way of constraining the PBH mass function [19].

${ }^{2}$ In the following, we use natural units for which $G=\hbar=c=k_{\mathrm{B}}=1$. 
In this formula, the most important component is the greybody factor which encodes the discrepancy between $\mathrm{BH}$ radiation and pure blackbody radiation. It is evaluated by evolving a plane wave radially from the $\mathrm{BH}$ horizon to the spatial infinity and comparing the amplitudes of the wave at these two extreme positions

$$
\Gamma_{i}\left(E, M, x_{j}\right) \equiv\left|\frac{Z_{\infty}}{Z_{\mathrm{hor}}}\right|^{2} .
$$

Hawking radiation yield thus depends on the spin of the emitted particle, and on the other internal degrees of freedom such as its charge and angular momentum. Couplings between the particle and the $\mathrm{BH}$ electric charges, or between the particle and the $\mathrm{BH}$ angular momentum [24-26] give rise to interesting phenomena such as superradiance [27]. Although numerical and analytical estimates existed for the greybody factors for various BH solutions ([28] is often cited), precise numerical computations of these factors was lacking in the literature.

We can deduce the time evolution of BHs by integrating over the energy and momentum loss

$$
\begin{aligned}
& f\left(M, a^{*}\right) \equiv-M^{2} \frac{\mathrm{d} M}{\mathrm{~d} t}=M^{2} \int_{0}^{+\infty} \sum_{i} \sum_{\text {dof. }} \frac{E}{2 \pi} \frac{\Gamma_{i}\left(E, M, a^{*}\right)}{e^{E^{\prime} / T} \pm 1} \mathrm{~d} E, \\
& g\left(M, a^{*}\right) \equiv-\frac{M}{a^{*}} \frac{\mathrm{d} J}{\mathrm{~d} t}=\frac{M}{a^{*}} \int_{0}^{+\infty} \sum_{i} \sum_{\text {dof. }} \frac{m}{2 \pi} \frac{\Gamma_{i}\left(E, M, a^{*}\right)}{e^{E^{\prime} / T} \pm 1} \mathrm{~d} E,
\end{aligned}
$$

where $J \equiv a^{*} M^{2}$ is the angular momentum of the BH and $m$ that of the particle. $f$ and $g$ can be interpreted as some renormalized number of (angular momentum) degrees of freedom emitted by BHs. These Page factors allow one to write differential equations for the mass and spin of the $\mathrm{BH}$

$$
\begin{aligned}
\frac{\mathrm{d} M}{\mathrm{~d} t} & =-\frac{f\left(M, a^{*}\right)}{M^{2}}, \\
\frac{\mathrm{d} a^{*}}{\mathrm{~d} t} & =\frac{a^{*}\left(2 f\left(M, a^{*}\right)-g\left(M, a^{*}\right)\right)}{M^{3}},
\end{aligned}
$$

where the mass evolution equation indeed shows the behaviour of Eq. (2) if $f\left(M, a^{*}\right)$ is taken as a constant. Indeed, for sufficiently light BHs $\left(M \lesssim 10^{10} \mathrm{~g}\right.$ ), all SM degrees of freedom are emitted and $f$ and $g$ do not evolve during evaporation. In any case, the Page factors vary at most of one order of magnitude during the evaporation of heavier BHs.

\section{BlackHawk content}

BlackHawk has been designed as a public tool to compute the Hawking radiation emitted by a variety of $\mathrm{BH}$ solutions, with any initial mass and spin distribution, and to provide spectra of final particles that can be compared to astrophysical observations ${ }^{3}$.

Various PBHs distributions taken from the literature have been implemented in BlackHawk, like the monochromatic one with a single $\mathrm{BH}$ mass and spin, and the log-normal one which is the result of $\mathrm{PBH}$ production by a narrow peak in the power spectrum of primordial density fluctuations. A lot of effort has been put into the computation of the greybody factors for Schwarzschild (nonrotating, uncharged) and Kerr (rotating, uncharged) BHs, which are per se interesting results. These

\footnotetext{
${ }^{3}$ Many more details can be found in the BlackHawk manual [23]
} 


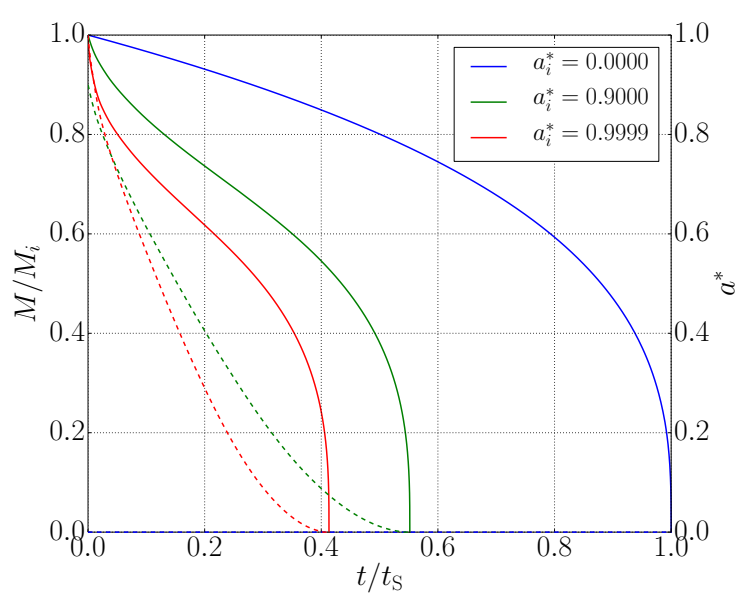

Figure 1: Evolution of a BH mass (solid) and spin (dashed) following Eqs. (6) and (7). Taken from [15].

greybody factors are used to compute the Page factors Eq. (6) and Eq. (7), which are then used to compute the mass and spin evolution of the distribution of BHs.

Hawking radiation is described in terms of Standard Model (SM) particle emission. Of course, any particle beyond the SM would be emitted by the BH horizon, such as any particle DM candidate, or the putative graviton (included in BlackHawk). On the other hand, astrophysical observations are based on the detection of stable particles, or at least on the imprints left in some cosmological era by stable particles at that timescale. Thus, particle physics codes (PYTHIA [29] and HERWIG [30]) are used to hadronize and decay the SM particles to stable ones ${ }^{4}$. In BlackHawk, they come in the form of tabulated transfer functions from primary SM particles to secondary stable particles.

\section{Primordial Black Hole detection and constraints}

BlackHawk is a versatile tool. The tabulated values of greybody factors, the Page factors, and the tabulated transfer functions between primary and secondary particles can be used outside the main code for e.g. BH evolution study [15] (see Fig. 1) or DM indirect searches. These transfer function tables were modeled on those of M. Cirelli et al. [31] (which were updated with QCD corrections in [32]). In BlackHawk we computed those tables from scratch using the latest available version of the particle physics codes. Of course, the main aim of BlackHawk is to provide spectra for constraining the abundance of $\mathrm{PBHs}$ in the universe, or for predicting signals emitted by known or putative astrophysical objects. Here we give a short overview of the work that has been done so far.

First, BlackHawk can predict the instantaneous spectrum of a population of PBHs in our Galaxy. If PBHs represent a substantial fraction or all of DM, they are expected to be distributed as $\mathrm{DM}$ in the galactic halo, following for example the Navarro-Frenk-White (NFW) profile given by [33]

$$
\rho_{\mathrm{PBH}}(r)=\rho_{\mathrm{DM}}(r) \propto \frac{1}{\left(r / r_{s}\right)(1+r / r s)^{2}},
$$

\footnotetext{
${ }^{4}$ Stable in the sense of cosmologically stable, or stable at the time of BBN for example.
} 
where $r_{s}$ is a characteristic radius. Then, if $\mathrm{PBHs}$ are monochromatic, i.e. with the same unique mass, it is easy to deduce the flux of particles of type $i$ incident on an Earth or space based detector resulting from the integration over the galactic bulk

$$
\frac{\mathrm{d} F_{i}}{\mathrm{~d} E}=\int \frac{\mathrm{d} \Omega}{4 \pi} \int \mathrm{d} l \frac{\rho_{\mathrm{PBH}}(l)}{M_{\mathrm{PBH}}} \frac{\mathrm{d}^{2} N_{i}}{\mathrm{~d} t \mathrm{~d} E},
$$

where $l$ is the line of sight coordinate. Of course, this flux depends on the precise distribution of DM, and deviations from NFW induce modified constraints/signals. For example, BlackHawk can predict the density of high-energy particles emitted by PBHs at the galactic center. These particles evolve in a strong magnetic field and thus produce synchrotron radiation in the radio band, that can be used to constrain the amount of PBHs [34]. PBHs of $M_{\mathrm{PBH}} \lesssim 10^{17} \mathrm{~g}$ emit electrons and positrons. The annihilation of the $e^{ \pm}$in the galactic center may contribute to the $511 \mathrm{keV}$ line measured in the direction of the galactic center [35]. On the same level, the INTEGRAL measure of the soft gamma-ray spectrum of the Galaxy sets constraints on the abundance of light PBHs $[36,37]$ and neutrino scintillators such as JUNO are sensitive to (anti)neutrinos [38, 39].

Second, BlackHawk can be used to constrain the cosmological amount of PBHs. If PBHs represent a substantial fraction of DM, then they can explain both the cosmological DM and galactic DM. For that, BlackHawk can compute the emission of particles throughout the whole BH lifetime, and integrating this emission, taking the cosmological redshift into account, gives access to the isotropic background flux of e.g. (soft) gamma-rays [40, 41]

$$
I \equiv E \frac{\mathrm{d} F_{\gamma}}{\mathrm{d} E}=\frac{1}{4 \pi} n_{\mathrm{PBH}}\left(t_{0}\right) E \int_{t_{\min }}^{t_{\max }}(1+z) \frac{\mathrm{d}^{2} N_{\gamma}}{\mathrm{d} t \mathrm{~d} E}((1+z) E) \mathrm{d} t,
$$

where $n_{\mathrm{PBH}}\left(t_{0}\right)$ is the number density of PBHs today, $z(t)$ is the redshift and $\mathrm{d}^{2} N_{\gamma} / \mathrm{d} t \mathrm{~d} E$ is the secondary spectrum of photons. The results of this computation, taking the PBH spin into account, are presented in Fig. 2. This prediction has been adapted to PBHs in scenarios with large extra spatial dimensions [42], opening an interesting window on the ability of BlackHawk to probe alternative gravitational theories.

Third, BlackHawk can be used together with Big-Bang nucleosynthesis (BBN) codes to provide constraints on very light PBHs that evaporate when the light elements are forged at the beginning of the universe. Indeed, energetic particles emitted by $M_{\mathrm{PBH}} \sim 10^{9}-10^{13} \mathrm{~g}$ PBHs can change the neutron to proton ratio at the onset of $\mathrm{BBN}$, induce nuclear reactions during $\mathrm{BBN}$ or photo-dissociate the final light elements after BBN. Hence, the modified Deuterium and/or Helium abundances can provide strong constraints on the abundance of light PBHs at the BBN epoch [43].

Fourth, BlackHawk can predict the signals emitted by astrophysical structures in terms of Hawking radiation in various energy bands. These constraints are independent from and complementary to the cosmological and galactic ones. For example, PBHs evaporating in nearby dwarf galaxies such as Leo T would deposit energy - heat and ionization - in such structures. Constraints can be set on the abundance of PBHs in these strongly DM-dominated objects [37, 44, 45]. More exotic is the prediction of the radio $(\gamma)$ and gravitational wave $(g)$ power emission of a putative Planet 9, would it be a $\mathrm{PBH}$ of $M_{\mathrm{P} 9} \sim 10 M_{\oplus}[46]$

$$
\frac{\mathrm{d}^{2} \mathcal{P}_{\gamma / g}}{\mathrm{~d} v \mathrm{~d} S}=\frac{1}{4 \pi r_{\mathrm{S}}^{2}} E \frac{\mathrm{d}^{2} N_{\gamma / g}}{\mathrm{~d} t \mathrm{~d} v},
$$




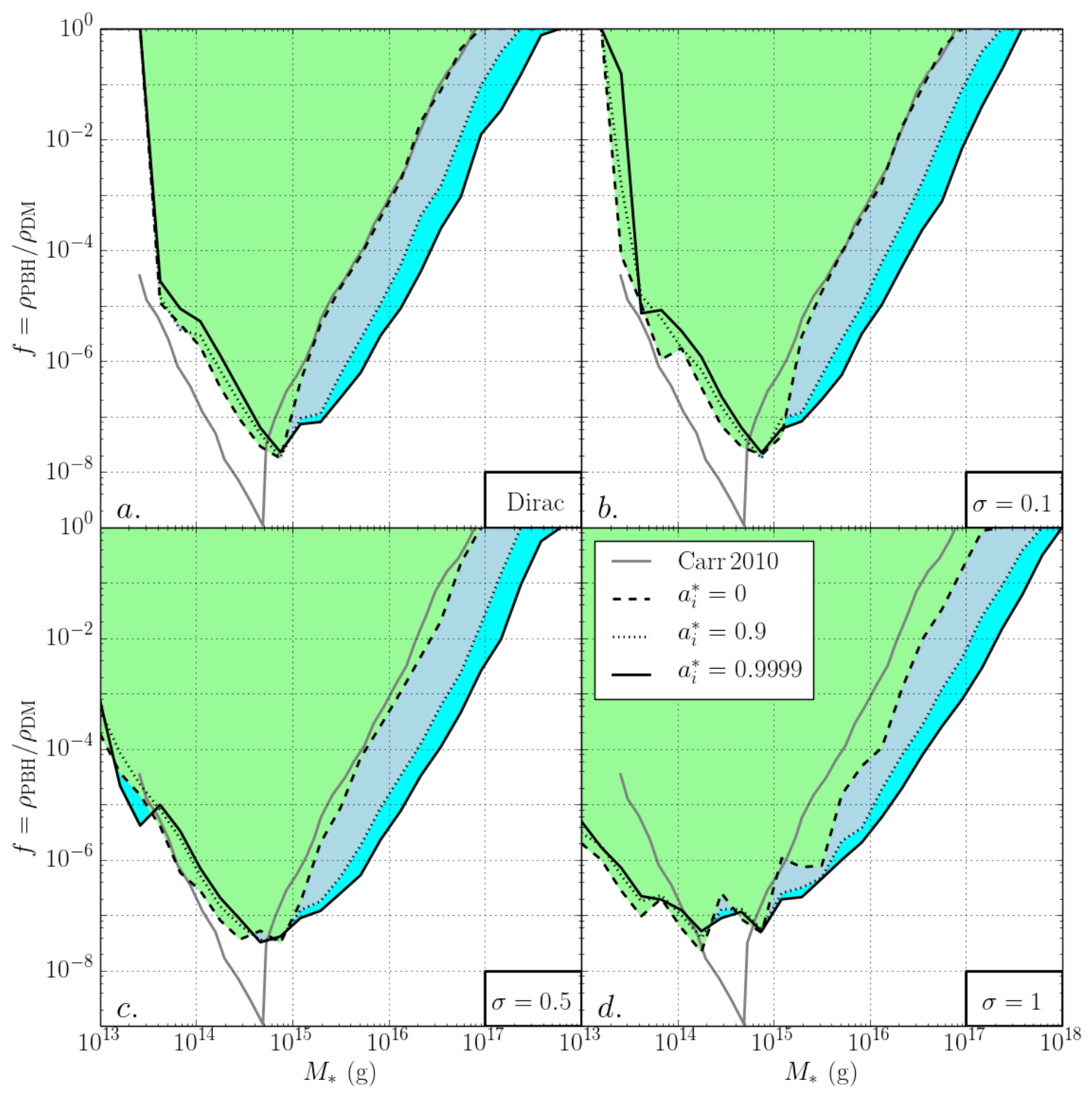

Figure 2: Constraints on the DM fraction composed of PBHs from the isotropic extragalactic gamma-ray background, where $\sigma$ (b, c, d) is the standard deviation of the PBH log-normal mass distribution, Dirac (a) corresponding to a monochromatic distribution. Taken from [41].

where $r_{\mathrm{S}}$ is the Planet 9 Schwarzschild radius and $v$ is the wave frequency. Prediction of the signal in terms of radio photons is given in Fig. 3.

Interestingly, most of these studies used the fact that BlackHawk allows the user to compute the spectra for realistic spinning BHs and extended mass functions. This is the main improvement of this public code over previous constraints, beside the more precise computation of the Hawking radiation rates. 


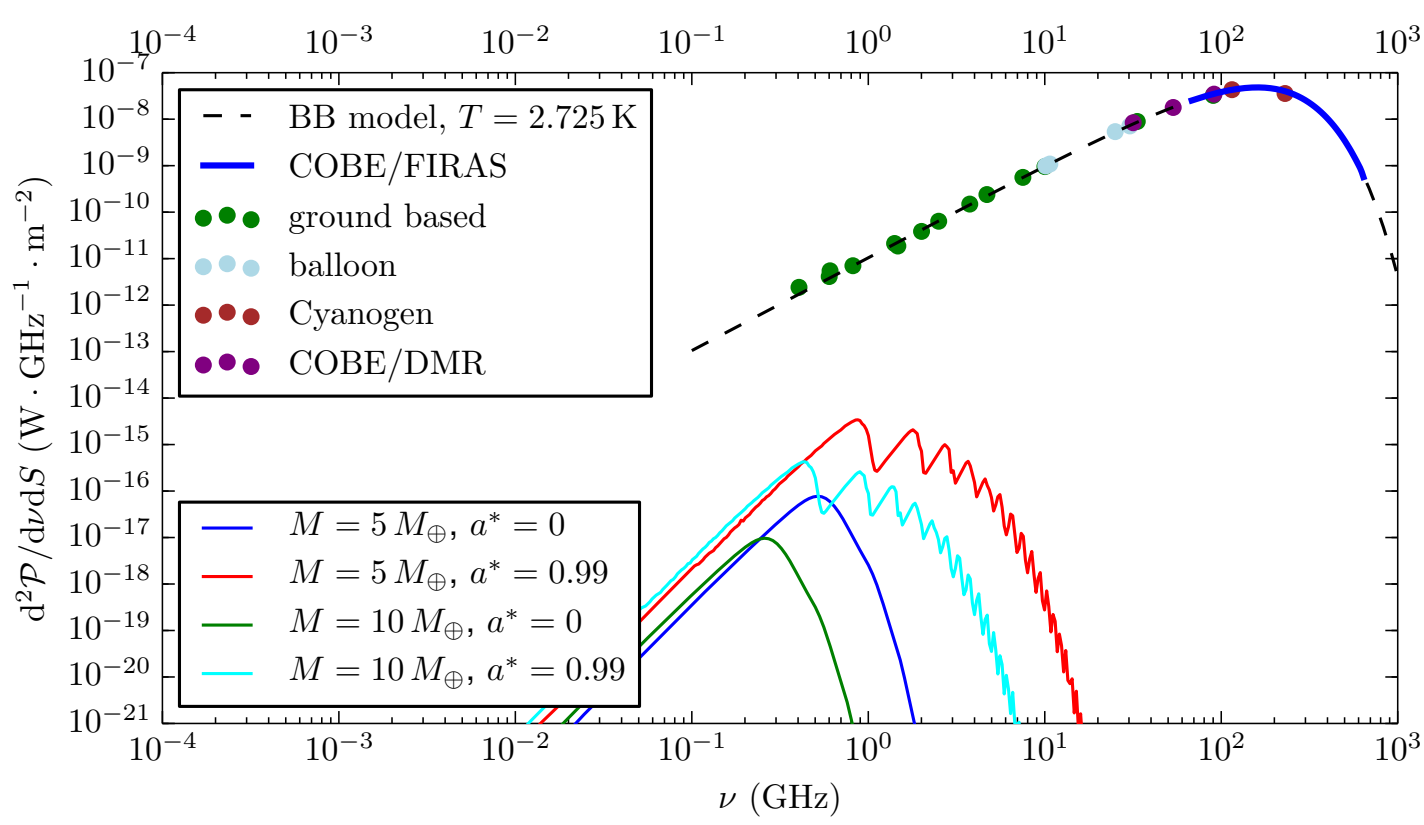

Figure 3: Comparison between the Planet 9 radio signal and the CMB background. Adapted from [46].

\section{Conclusion}

We have summarized here the context of development of the public code BlackHawk, which provides a powerful and versatile tool to compute the Hawking radiation spectra of any distribution of BHs. We have described the basics of Hawking radiation and the main capabilities of BlackHawk and given an overview of the studies this code has already helped to pursue. BlackHawk is not a fixed entity and receives regular updates to correct bugs and improve computation time and user friendliness. Moreover, we have already invested work towards a major update that will enhance its capabilities: new BH solutions as well as a better low-energy treatment and additional particles will be added in the near future.

\section{Acknowledgments}

We would like to thank the organizers of the TOOLS 2020 conference for giving us the opportunity to present our work.

\section{References}

[1] C. Tao, Dark Matter searches: an overview, Journal of Instrumentation 15 (2020) C06054.

[2] C. Pérez de los Heros, Status, Challenges and Directions in Indirect Dark Matter Searches, arXiv e-prints (2020) arXiv:2008.11561 [2008.11561].

[3] Y.B. Zel'dovich and I.D. Novikov, The Hypothesis of Cores Retarded during Expansion and the Hot Cosmological Model, Soviet Astronomy 10 (1967) 602. 
[4] LIGO Scientific Collaboration and Virgo Collaboration, Binary Black Hole Population Properties Inferred from the First and Second Observing Runs of Advanced LIGO and Advanced Virgo, Astrophys. J. Lett. 882 (2019) L24 [1811. 12940].

[5] Event Horizon Telescope Collaboration, K. Akiyama et al., First M87 Event Horizon Telescope Results. I. The Shadow of the Supermassive Black Hole, Astrophys. J. Lett. 875 (2019) L1 [1906. 11238].

[6] B. Carr, K. Kohri, Y. Sendouda and J. Yokoyama, Constraints on Primordial Black Holes, arXiv e-prints (2020) arXiv:2002.12778 [2002 . 12778].

[7] A.M. Green and B.J. Kavanagh, Primordial Black Holes as a dark matter candidate, arXiv e-prints (2020) arXiv:2007.10722 [2007.10722].

[8] E. Khalouei, H. Ghodsi, S. Rahvar and J. Abedi, Possibility of Primordial Black holes as the source of gravitational wave events in the advanced LIGO detector, arXiv e-prints (2020) arXiv:2011.02772 [2011.02772].

[9] C. Boehm, A. Kobakhidze, C.A.J. O'Hare, Z.S.C. Picker and M. Sakellariadou, Eliminating the LIGO bounds on primordial black hole dark matter, arXiv e-prints (2020) arXiv:2008.10743 [2008. 10743].

[10] J.L.G. Sobrinho and P. Augusto, Stellar mass primordial black holes as cold dark matter, MNRAS 496 (2020) 60 [2005 . 10037].

[11] K.W.K. Wong, G. Franciolini, V. De Luca, V. Baibhav, E. Berti, P. Pani et al., Constraining the primordial black hole scenario with Bayesian inference and machine learning: the GWTC-2 gravitational wave catalog, arXiv e-prints (2020) arXiv:2011.01865 [2011.01865].

[12] J. Garcia-Bellido, J.F. Nuño Siles and E. Ruiz Morales, Bayesian analysis of the spin distribution of LIGO/Virgo black holes, arXiv e-prints (2020) arXiv:2010.13811 [2010.13811].

[13] O. Burke, J.R. Gair, J. Simón and M.C. Edwards, Extreme precision for extreme spin: probing near-extremal black holes using LISA, arXiv e-prints (2020) arXiv:2010.05932 [2010.05932].

[14] V. De Luca, G. Franciolini, P. Pani and A. Riotto, The evolution of primordial black holes and their final observable spins, JCAP 2020 (2020) 052 [2003. 02778].

[15] A. Arbey, J. Auffinger and J. Silk, Evolution of primordial black hole spin due to Hawking radiation, MNRAS 494 (2020) 1257 [1906. 04196].

[16] S.W. Hawking, Particle creation by black holes, Communications in Mathematical Physics 43 (1975) 199.

[17] S.W. Hawking, Black hole explosions?, Nature 248 (1974) 30. 
[18] B. Carr, F. Kuhnel and L. Visinelli, Black Holes and WIMPs: All or Nothing or Something Else, arXiv e-prints (2020) arXiv:2011.01930 [2011.01930].

[19] D.-C. Dai, R. Gregory and D. Stojkovic, Connecting the Higgs potential and primordial black holes, Phys. Rev. D 101 (2020) 125012 [1909.00773].

[20] R. Dong, W.H. Kinney and D. Stojkovic, Gravitational wave production by Hawking radiation from rotating primordial black holes, JCAP 2016 (2016) 034 [1511.05642].

[21] K. Inomata, M. Kawasaki, K. Mukaida, T. Terada and T.T. Yanagida, Gravitational wave production right after a primordial black hole evaporation, Phys. Rev. D 101 (2020) 123533 [2003.10455].

[22] D. Hooper, G. Krnjaic, J. March-Russell, S.D. McDermott and R. Petrossian-Byrne, Hot Gravitons and Gravitational Waves From Kerr Black Holes in the Early Universe, arXiv e-prints (2020) arXiv:2004.00618 [2004.00618].

[23] A. Arbey and J. Auffinger, BlackHawk: a public code for calculating the Hawking evaporation spectra of any black hole distribution, European Physical Journal C 79 (2019) 693 [1905. 04268].

[24] D.N. Page, Particle emission rates from a black hole: Massless particles from an uncharged, nonrotating hole, Phys. Rev. D 13 (1976) 198.

[25] D.N. Page, Particle emission rates from a black hole. II. Massless particles from a rotating hole, Phys. Rev. D 14 (1976) 3260.

[26] D.N. Page, Particle emission rates from a black hole. III. Charged leptons from a nonrotating hole, Phys. Rev. D 16 (1977) 2402.

[27] R. Brito, V. Cardoso and P. Pani, Superradiance: New Frontiers in Black Hole Physics, vol. 906, Springer (2015), [1501.06570].

[28] J.H. MacGibbon and B.R. Webber, Quark- and gluon-jet emission from primordial black holes: The instantaneous spectra, Phys. Rev. D 41 (1990) 3052.

[29] T. Sjöstrand, S. Ask, J.R. Christiansen, R. Corke, N. Desai, P. Ilten et al., An introduction to PYTHIA 8.2, Computer Physics Communications 191 (2015) 159 [1410.3012].

[30] J. Bellm, G. Bewick, S. Ferrario Ravasio, S. Gieseke, D. Grellscheid, P. Kirchgaeßer et al., Herwig 7.2 release note, European Physical Journal C 80 (2020) 452 [1912 . 06509].

[31] M. Cirelli, G. Corcella, A. Hektor, G. Hütsi, M. Kadastik, P. Panci et al., PPPC 4 DM ID: $a$ poor particle physicist cookbook for dark matter indirect detection, JCAP 2011 (2011) 051 [1012.4515].

[32] S. Amoroso, S. Caron, A. Jueid, R. Ruiz de Austri and P. Skands, Estimating QCD uncertainties in Monte Carlo event generators for gamma-ray dark matter searches, JCAP 2019 (2019) 007 [1812.07424]. 
[33] J.F. Navarro, C.S. Frenk and S.D.M. White, The Structure of Cold Dark Matter Halos, Astrophys. J. 462 (1996) 563 [astro-ph/9508025].

[34] M.H. Chan and C.M. Lee, Constraining primordial black hole fraction at the galactic centre using radio observational data, MNRAS 497 (2020) 1212 [2007.05677].

[35] R. Laha, Primordial Black Holes as a Dark Matter Candidate Are Severely Constrained by the Galactic Center 511 keV $\gamma$-Ray Line, Phys. Rev. Lett. 123 (2019) 251101 [1906. 09994].

[36] R. Laha, J.B. Muñoz and T.R. Slatyer, I N T E G R A L constraints on primordial black holes and particle dark matter, Phys. Rev. D 101 (2020) 123514 [2004 . 00627].

[37] R. Laha, P. Lu and V. Takhistov, Gas Heating from Spinning and Non-Spinning Evaporating Primordial Black Holes, arXiv e-prints (2020) arXiv:2009.11837 [2009 . 11837].

[38] B. Dasgupta, R. Laha and A. Ray, Neutrino and Positron Constraints on Spinning Primordial Black Hole Dark Matter, Phys. Rev. Lett. 125 (2020) 101101 [1912.01014].

[39] S. Wang, D.-M. Xia, X. Zhang, S. Zhou and Z. Chang, Constraining the Primordial Black Holes as Dark Matter at JUNO, arXiv e-prints (2020) arXiv:2010.16053 [2010. 16053].

[40] G. Ballesteros, J. Coronado-Blázquez and D. Gaggero, X-ray and gamma-ray limits on the primordial black hole abundance from Hawking radiation, Physics Letters B 808 (2020) 135624 [1906. 10113].

[41] A. Arbey, J. Auffinger and J. Silk, Constraining primordial black hole masses with the isotropic gamma ray background, Phys. Rev. D 101 (2020) 023010 [1906. 04750].

[42] G. Johnson, Primordial black hole constraints with large extra dimensions, JCAP 2020 (2020) 046 [2005 . 07467].

[43] Y. Luo, C. Chen, M. Kusakabe and T. Kajino, Impacts of Hawking Radiation from Primordial Black Holes in Critical Collapse Model on the Light Element Abundances, arXiv e-prints (2020) arXiv:2011.10937 [2011.10937].

[44] H. Kim, A constraint on light primordial black holes from the interstellar medium temperature, arXiv e-prints (2020) arXiv:2007.07739 [2007.07739].

[45] A. Coogan, L. Morrison and S. Profumo, Direct Detection of Hawking Radiation from Asteroid-Mass Primordial Black Holes, arXiv e-prints (2020) arXiv:2010.04797 [2010.04797].

[46] A. Arbey and J. Auffinger, Detecting Planet 9 via Hawking radiation, arXiv e-prints (2020) arXiv:2006.02944 [2006.02944]. 